hep-th/9512126, SHEP 95/40

\title{
On Non-Abelian Duality in Sigma Models
}

\author{
by \\ Noureddine Mohammedi f \\ Department of Physics \\ University of Southampton \\ Southampton SO17 1BJ \\ U.K.
}

\begin{abstract}
A method for implementing non-Abelian duality on string backgrounds is given. It is shown that a direct generalisation of the familiar Abelian duality induces an extra local symmetry in the gauge invariant theory. The non-Abelian isometry group is shown to be enlarged to a non-semi-simple group. However, upon eliminating the gauge fields to obtain the dual theory the new algebra does not close. Therefore the gauge fixing procedure becomes problematic. The new method proposed here avoids these issues and leads to a dual theory in the proper sense of duality.
\end{abstract}

\footnotetext{
${ }^{1}$ e-mail: nouri@hep.phys.soton.ac.uk
} 


\section{Introduction}

Target space duality has been the focus of many investigations in the last decade (see [1] for a review and further references). This is due to its relevance to the understanding of the structure of the string backgrounds (or moduli space) on which compactification takes place. This duality connects two conformal backgrounds which are, a priori, totally different. The simplest examples of this duality are the $O(d, d, R)$ Narain [2] transformation relating all possible toroidal compactifications in $d$ dimensions. Generalisations of Narain's transformations have also been found for backgrounds possessing $d$ Abelian isometries [0]. This type of duality is refered to as the Abelian duality. The duality is non-Abelian if the backgrounds isometries are non-Abelian.

The Abelian duality is better understood at the level of the Lagrangian defining the low energy of string theoryt [5]. The dual non-linear sigma model is obtained by gauging the $U(1)$ isometries of this Lagrangian and at the same time constraining, by means of Lagrange multipliers, the field strength of each $U(1)$ gauge field to vanish. Integrating out these Lagrange multipliers and fixing the gauge invariance yields the original model. On the other hand, integrating over the gauge fields and keeping the Lagrange multipliers results in the dual sigma model. If the original backgrounds are conformal backgrounds (i.e. they satisfy the vanishing of the beta functions conditions) then the dual backgrounds are also conformal and form consistent backgrounds on which the string propagates [6].

The generalisation of the above construction to backgrounds possessing non-Abelian isometries could, in principle, be carried out in exact analogy with the Abelian case [7]. However, this straightforward generalisations proves to be problematic [8] In particular, the dual backgrounds do not, in general, satisfy the vanishing of the beta functions conditions.

In this letter we show that the Lagrange multiplier term needed for non-Abelian duality cause the dual Lagrangian to have an extra symmetry which, when combined with the nonAbelian isometry group, forms a non-semi-simple closed algebra. This new algebra does not close, however, when the gauge fields are integrated out. This fact makes gauge fixing difficult.

This new symmetry arise because the Lagrange multipliers carry now internal indices. A similar symmetry has appeared in the context of gauge theories in four dimensions [9]. The method we propose for non-Abelian duality uses Lagrange multiplier carrying no gauge group indices. The implementation of this method is a natural one which stems from the

\footnotetext{
${ }^{2}$ Duality has also been treated in terms of canonical transformations [ $[4]$.
} 
mathematical formalism of gauging isometries in a general non-linear sigma model [10, 11].

We start, in section one, by stating the general formalism of gauging isometries in nonlinear sigma model. The usual way of finding the dual theory is also explained. This is in direct analogy with the Abelian case. The new symmetry is revealed in section three and the new method is given.

\section{The Abelian analogy}

We start this section by giving a summary of gauging Abelian and non-Abelian isometries in a general sigma model. The action for the general (ungauged) bosonic two-dimensional non-linear sigma model is given by

$$
S(\varphi)=\int \mathrm{d}^{2} x \sqrt{\gamma}\left(\gamma^{\mu \nu} g_{i j}(\varphi) \partial_{\mu} \varphi^{i} \partial_{\nu} \varphi^{j}+\widehat{\epsilon}^{\mu \nu} b_{i j}(\varphi) \partial_{\mu} \varphi^{i} \partial_{\nu} \varphi^{j}+D(\varphi) R^{(2)}\right) .
$$

In this equation $\gamma_{\mu \nu}$ is the metric on the two-dimensional world sheet, $\gamma$ is its determinant, $R^{(2)}$ is the scalar curvature and $\widehat{\epsilon}^{\mu \nu}=\epsilon^{\mu \nu} / \sqrt{\gamma}$ is the alternating tensor. The metric $g_{i j}$, the anti-symmetric tensor $b_{i j}$ and the dilaton field $D$ correspond to the set of massless modes of the associated string theory.

The sigma model Lagrangian is manifestly invariant under global reparametrisation of the target space. However, the only global symmetries suitable for gauging are those for which the metric remains form invariant. Such symmetries form a Lie group $G$, the isometry group of the metric. A general infinitesimal global isometry is given by the transformation

$$
\delta \varphi^{i}=\alpha^{a} K_{a}^{i}(\varphi)
$$

The generator of this transformation are $K_{a}=K_{a}^{i} \partial_{i}$ and they satisfy the Lie algebra of $G$

$$
\left[K_{a}, K_{b}\right]=f_{a b}^{c} K_{c} \quad \text { or } \quad K_{a}^{i} \partial_{i} K_{b}^{j}-K_{b}^{i} \partial_{i} K_{a}^{j}=f_{a b}^{c} K_{c}^{j}
$$

where $f_{b c}^{a}$ are field-independent structure constants.

The procedure for obtaining the dual sigma model consists in gauging the above global symmetry and constraining the gauge field strength to vanish through the addition of a Lagrange multiplier. It is straightforward to gauge the first term in the Lagrangian. This is achieved through the familiar mininal coupling

$$
\partial_{\mu} \varphi^{i} \quad \longrightarrow \quad D_{\mu}=\partial_{\mu} \varphi^{i}+A^{a} K_{a}^{i}
$$

and requiring that under (2), with $\alpha^{a}$ now a local function,

$$
\delta A_{\mu}^{a}=-\partial_{\mu} \alpha^{a}-f_{b c}^{a} \alpha^{b} A_{\mu}^{c}
$$


where $K_{a}^{i}$ satisfy the Killing vector condition

$$
K_{a}^{k} \partial_{k} g_{i j}+g_{k j} \partial_{i} K_{a}^{k}+g_{i k} \partial_{j} K_{a}^{k}=0
$$

Gauging the second term of the Lagrangian requires, however, a special treatment. Under the global isometry transformation, this term is invariant provided that

$$
K_{a}^{k} \partial_{k} b_{i j}+b_{k j} \partial_{i} K_{a}^{k}+b_{i k} \partial_{j} K_{a}^{k}=\partial_{i} L_{a j}-\partial_{j} L_{a i}
$$

for some vector $L_{a i}$. Because of this last equation, the minimal coupling used to gauge the first term does not lead to a gauge invariant action. The gauged invariant theory is found through the use of Noether method and we have [10, 11]

$$
\begin{aligned}
S(\varphi, A) & =\int \mathrm{d}^{2} x \sqrt{\gamma}\left[\gamma^{\mu \nu} g_{i j} D_{\mu} \varphi^{i} D_{\nu} \varphi^{j}+\widehat{\epsilon}^{\mu \nu}\left(b_{i j} \partial_{\mu} \varphi^{i} \partial_{\nu} \varphi^{j}-2 A_{\mu}^{a} c_{a i} \partial_{\nu} \varphi^{i}-d_{a b} A_{\mu}^{a} A_{\nu}^{b}\right)\right. \\
& \left.+D(\varphi) R^{(2)}\right]
\end{aligned}
$$

with $d_{a b}$ anti-symmetric and where

$$
\begin{aligned}
c_{a i} & =b_{i j} K_{a}^{j}+L_{a i} \\
d_{a b} & =c_{i a} K_{b}^{i} .
\end{aligned}
$$

The action is then gauge invariant provided that

$$
\begin{aligned}
K_{b}^{j} \partial_{j} c_{a i}+c_{a j} \partial_{i} K_{b}^{j} & =-f_{a b}^{e} c_{e i} \\
K_{c}^{i} \partial_{i} d_{a b} & =f_{c a}^{e} d_{e b}-f_{c b}^{e} d_{e a}
\end{aligned}
$$

with $L_{a i}$ as defined in (7). There are other ways of writing the gauge invariance conditions showing explicitly the anti-symmetry of $d_{a b}$ and involving only $L_{a i}$ [10].

Finally, the dilaton term is gauge invariant provided that $K_{a}^{i} \partial_{i} D=0$.

If one follows the analogy with the Abelian duality then one considers the following action [7]

$$
S(\varphi, A, \lambda)=S(\varphi, A)+\int \mathrm{d}^{2} x \sqrt{\gamma} \widehat{\epsilon}^{\mu \nu} F_{\mu \nu}^{a} \lambda_{a},
$$

where $F_{\mu \nu}^{a}=\partial_{\mu} A_{\nu}^{a}-\partial_{\nu} A_{\mu}^{a}-f_{b c}^{a} A_{\mu}^{b} A_{\nu}^{c}$ and for gauge invariance the Lagrange multiplier $\lambda_{a}$ transforms as

$$
\delta \lambda_{a}=-f_{a b}^{c} \alpha^{b} \lambda_{c}
$$

Integrating over the Lagrange multiplier $\lambda_{a}$ leads to $F_{\mu \nu}^{a}=0$ which is solved by $A_{\mu}^{a}=$ $e_{i}^{a}(X) \partial_{\mu} X^{i}$, where $e_{i}^{a}(X)$ are vielbeins satisfying the Maurer-Cartan conditions. Choosing 
a gauge in which $A_{\mu}^{a}=0$ leads then to the original action $S(\varphi)$. Now keeping the Lagrange multiplier and integrating out the gauge fields, instead, leads to the dual theory. The equations of motion for the gauge field (which is the same as doing the Gaussian integral) is

$$
A_{\rho}^{c}=\widetilde{M}_{\rho \mu}^{c a}\left[\left(\widehat{\epsilon}^{\mu \nu} c_{a i}-\gamma^{\mu \nu} g_{i j} K_{a}^{j}\right) \partial_{\nu} \varphi^{i}-\widehat{\epsilon}^{\mu \nu} \partial_{\nu} \lambda_{a}\right]
$$

where $\widetilde{M}_{a b}^{\mu \nu}$ is defined by

$$
\begin{aligned}
M_{a b}^{\mu \nu} & =\gamma^{\mu \nu} g_{i j} K_{a}^{i} K_{b}^{j}-\widehat{\epsilon}^{\mu \nu}\left(d_{a b}+f_{a b}^{e} \lambda_{e}\right) \\
M_{a b}^{\mu \nu} \widetilde{M}_{\nu \rho}^{b c} & =\delta_{\rho}^{\mu} \delta_{c}^{a} .
\end{aligned}
$$

Substituting for $A_{\mu}^{a}$ in (11) we get the following action

$$
\begin{aligned}
S(\varphi, \lambda) & =S(\varphi)+\int \mathrm{d}^{2} x \sqrt{\gamma}\left\{-\widetilde{M}_{\beta \alpha}^{a d}\left(\widehat{\epsilon}^{\alpha \mu} \widehat{\epsilon}^{\beta \nu} c_{d i} c_{a j}+\gamma^{\alpha \mu} \gamma^{\beta \nu} g_{i l} g_{j k} K_{d}^{l} K_{a}^{k}\right.\right. \\
& \left.-2 \widehat{\epsilon}^{\alpha \mu} \gamma^{\beta \nu} c_{d i} g_{j k} K_{a}^{k}\right) \partial_{\mu} \varphi^{i} \partial_{\nu} \varphi^{j}-\widetilde{M}_{\sigma \rho}^{a c} \widehat{\gamma}^{\sigma \nu} \widehat{\epsilon}^{\rho \mu} \partial_{\nu} \lambda_{a} \partial_{\mu} \lambda_{c} \\
& \left.+2 \widetilde{M}_{\rho \alpha}^{a d}\left(\widehat{\epsilon}^{\rho \nu} \widehat{\epsilon}^{\alpha \mu} c_{d i}-\widehat{\epsilon}^{\alpha \nu} \gamma^{\rho \nu} g_{i j} K_{d}^{j}\right) \partial_{\nu} \lambda_{a} \partial_{\mu} \varphi^{i}\right\}
\end{aligned}
$$

Naively one would say that there are $\operatorname{dim}(G)$ gauge parameters $\alpha^{a}$ which would be used to eliminate $\operatorname{dim}(G)$ fields from this action and one would then obtain a dual sigma model having the same number of fields as the original theory. However, the action $S(\varphi, A, \lambda)$ as given in (11) has in fact an extra symmetry as shown below.

\section{The new method}

It is easy to see that by anti-symmetry the action (11) is, in addition to its gauge invariance, also invariant under the local transformations

$$
\begin{aligned}
\widetilde{\delta} \lambda_{a} & =\widehat{\epsilon}^{\mu \nu} f_{a b}^{e} \xi_{e} F_{\mu \nu}^{b} \\
\widetilde{\delta} \varphi^{i} & =\widetilde{\delta} A_{\mu}^{a}=0
\end{aligned}
$$

where $\xi_{a}$ is the local gauge parameter. Therefore the gauge invariance group has been enlarged due to the addition of the Lagrange multiplier. The two local gauge transformation $\delta_{\alpha}$ and $\widetilde{\delta}_{\xi}$ form the following closed algebra

$$
\begin{aligned}
& {\left[\delta_{\alpha}, \delta_{\beta}\right]=\delta_{\gamma}, \quad\left[\delta_{\alpha}, \widetilde{\delta}_{\omega}\right]=\widetilde{\delta}_{\rho}} \\
& {\left[\widetilde{\delta}_{\alpha}, \widetilde{\delta}_{\beta}\right]=0}
\end{aligned}
$$

with $\gamma^{a}=f_{b c}^{a} \alpha^{b} \beta^{c}$ and $\rho_{a}=f_{a b}^{c} \alpha^{b} \omega_{c}$. 
After eliminating the gauge fields by their equations of motion this is still a symmetry of $S(\varphi, \lambda)$ where $F_{\mu \nu}^{a}$ is now the field strength corresponding to $A_{\mu}^{a}$ as given in (13). We have explicitly checked that this is indeed the case. However the elimination of the gauge fields causes the above algebra not to close. This is a familiar issue in supergravity theories. Therefore one does not know how to fix the gauge invariance of the action $S(\varphi, \lambda)$.

One way out of this problem is to consider, instead of (11), the following Lagrange multiplier term

$$
I(\varphi, A, \lambda)=S(\varphi, A)+\int \mathrm{d}^{2} x \sqrt{\gamma} \widehat{\epsilon}^{\mu \nu} F_{\mu \nu}^{a} \Gamma_{a} \lambda .
$$

The Lagrange multiplier $\lambda$ does not carry now a gauge index and the extra gauge symmetry found previously is not present $(\delta \lambda=\widetilde{\delta} \lambda=0)$. The new quantity $\Gamma_{a}(\varphi)$ is a function which satisfies

$$
K_{a}^{i} \partial_{i} \Gamma_{b}=f_{a b}^{c} \Gamma_{c}
$$

This equation is necessary for gauge invariance. This Lagrange multiplier term reduces to the usual one when considering the Abelian case.

Another property of the the new Lagrange multiplier term can be seen by performing an integration by parts in (18)

$$
I(\varphi, A, \lambda)=S(\varphi, A)+\int \mathrm{d}^{2} x \sqrt{\gamma} \widehat{\epsilon}^{\mu \nu}\left(-2 \Gamma_{a} A_{\nu}^{a} \partial_{\mu} \lambda-2 \lambda A_{\nu}^{a} \partial_{i} \Gamma_{a} \partial_{\mu} \varphi^{i}-\lambda f_{b c}^{a} \Gamma_{a} A_{\mu}^{b} A_{\nu}^{c}\right) .
$$

The second and third terms of the Lagrange multiplier term can be simply absorbed by shifting both $c_{a i}$ and $d_{a b}$ in the following manner

$$
c_{a i} \longrightarrow c_{a i}-\lambda \partial_{i} \Gamma_{a}, d_{a b} \longrightarrow d_{a b}-\lambda f_{a b}^{c} \Gamma_{c}
$$

This shift maintains the necessary conditions for gauge invariance given in (10). Hence the only term in the Lagrange multiplier term which is a genuine Lagrange multiplier is

$$
I(\varphi, A, \lambda)=\widetilde{S}(\varphi, A)-2 \int \mathrm{d}^{2} x \sqrt{\gamma} \widehat{\epsilon}^{\mu \nu} \Gamma_{a} A_{\nu}^{a} \partial \lambda_{\mu},
$$

where $\widetilde{S}(\varphi, A)$ is obtained from $S(\varphi, A)$ by replacing $c_{a i}$ and $d_{a b}$ by their shifted values (21).

Written in this form, the dual model has a global $U(1)$ symmetry acting on $\lambda$ only

$$
\lambda \longrightarrow \lambda+\omega
$$

with $\omega$ a constant. This transformation must be accompanied by the corresponding shifts in $c_{a i}$ and $d_{a b}$. It can be easily shown that the freedom in shifting these two quantities stems from a symmetry in the defining equation of $L_{a i}$ in (7), namely $L_{a i} \longrightarrow L_{a i}+\partial_{i} V_{a}$. 
If we now make an Abelian duality with respect to this symmetry we recover the original model. Gauging this $U(1)$ symmetry via the introduction of a $U(1)$ gauge field $B_{\mu}$ and adding a second Lagrange multiplier $\kappa$ we obtain

$$
I(\varphi, \lambda, \kappa, A, B)=S(\varphi, A, \lambda)+2 \int \mathrm{d}^{2} x \sqrt{\gamma} \widehat{\epsilon}^{\mu \nu}\left(-\Gamma_{a} A_{\nu}^{a} B_{\mu}+\kappa \partial_{\mu} B_{\nu}\right)
$$

Integrating out $B_{\mu}$ gives

$$
\Gamma_{a} A_{\mu}^{a}=\partial_{\mu} \kappa
$$

A gauge choice can now be made to obtain $A_{\mu}^{a}$ which would lead to $F_{\mu \nu}^{a}=0$ and the original model is thus recovered. Therefore in this method the dual of the dual theory is the original theory as in the Abelian case.

The procedure for eliminating the gauge fields from (18) follows steps similar to those in section two. The dual action is obtained by simply replacing in (15) $\lambda_{a}$ by $\psi_{a}=\lambda \Gamma_{a}$. The dual theory is then obtained by eliminating the extra degrees of freedom by gauge fixing. This procedure gives the dual target space metric and the dual anti-symmetric tensor field. The dual dilaton field is then given by the original dilaton field plus the contributions arising from a proper consideration of the path integral. Further details will be given elsewhere [13].

\section{Conclusions}

We have shown how a direct generalisation of the methods of Abelian duality to non-Abelian duality leads to the generation of a new local symmetry. This symmetry was not taken into account in previous works on non-Abelian duality. Due to this new symmetry, the isometry group is automatically promoted to a larger non-semi-simple group.

The fact that the dual backgrounds found in previous works did not satisfy the conformal invariance conditions (the vanishing of the beta functions) is due to not having taken into account this extra symmetry. After eliminating the gauge fields in order to obtain the dual theory, the new algebra no longer closes. This is a major issue when dealing with gauge theories. A treatment à la Batatin-Vilkovisky using the master equation might be of help [12. This is certainly worth investigating.

The method we propose does not allow the Lagrange multipliers to carry any internal indices. These internal indices are at the heart of the extra symmetry. Furthermore it was shown that in this method the dual of the dual theory is the original theory as expected.

Explicit and concrete examples where this new non-Abelian duality is constructed will be reported elsewhere [13]. Another issue that needs clarifications regards the global properties 
of the dual theory. The way we obtained the dual theory suggests that there must be connections between the global properties of the anti-symmetric tensor field and those of the Lagrange multipliers. Finally it would be desirable to see whether this duality could be explained in terms canonical transformations 4 .

Acknowledgements: I would like to thank Peter Hodges, Tim Morris and Douglas Ross for discussions.

\section{References}

[1] A. Giveon, M. Porrati and E. Rabinovici, Phys. Rep. 244 (1994) 77;

E. Alvarez, L. Alvarez-Gaumé and Y. Lozano, An Introduction to T-Duality in String Theory, hep-th/9410237.

[2] K. S. Narain, Phys. Lett. B169 (1986) 41;

K. S. Narain, M. H. Sarmadi and E. Witten, Nucl. Phys. B279 (1987) 369.

[3] A. Sen, Phys. Lett. B271 (1991) 295;

S. F. Hassan and A. Sen, Nucl. Phys. B375 (1992) 103;

J. Maharana and and J. H. Schwarz, Non Compact Symmetries in String Theory, Caltech preprint, CALT-68-1790 (1992);

A. Giveon and M. Roček, Nucl. Phys. B380 (1992) 128;

E. Kiritsis, Nucl. Phys. B405 (1993) 109;

A. Giveon and E. Kiritsis, Nucl. Phys. B411 (1994) 487;

G. Veneziano, Phys. Lett. B265 (1991);

A.A. Tseytlin, Mod. Phys. Lett. A6 (1991) 1721;

K. A. Meissner and G. veneziano, Phys. Lett. B267 (1991) 33; Mod. Phys. Lett. A6 (1991) 3397;

M. Gasperini and G. Veneziano, Phys. Lett. B277 (1992);

A. S. Schwarz and A. A. Tseytlin, Nucl. Phys. 399 (1993) 691.

[4] E. Alvarez, L. Alvarez-Gaumé, J.L.F. Barbón and Y. Lozano, Nucl. Phys. B415 (1994) 71 ;

Y. Lozano, Non-Abelian Duality and Canonical transformations, preprint PUPT-1532, hep-th/9503045;

T. Curtright and C. Zachos, Canonical Nonabelian Dual Transformations in Supersymmetric Field Theories, hep-th/95021126; Phys. Rev. D49 (1994) 5408.

[5] M. Roček and E. Verlinde, Nucl. Phys. 373 (1992) 630. 
[6] T. H. Buscher, Phys. Lett. B201 (1988) 466;

B. E. Fridling and A. Jevicki, Phys. Lett. B134 (1984) 70;

E. S. Fradkin and A. A. Tseytlin, Ann. Phys. B162 (1985) 31.

[7] X. de la Ossa and F. Quevedo, Nucl. Phys. B403 (1993) 377.

[8] A. Giveon and M. Roček, Nucl. Phys. B421 (1994) 173;

M. Gasperini, R. Ricci and G. Veneziano, Phys. Lett. B319 (1993) 438;

S. Elitzur, A. Giveon, E. Rabinovici, A. Schwimmer and G. Veneziano, Remarks on Non-abelian duality, hep-th/9409011;

C. Klimcík and P. Severa, Dual Non-abelian Duality and the Drinfeld Double, hepth/9502122;

O. Alvarez, Classical Geometry and Target Space Duality, hep-th/9511024.

[9] N. Mohammedi, Classical Duality in Gauge Theories, Southampton preprint, SHEP 95/23, hep-th/9507055.

[10] I. Jack, D. R. T. Jones, N. Mohammedi and H. Osborn, Nucl. Phys. 332 (1990) 359.

[11] C. M. Hull and B. Spence, Phys. Lett. B232 (1989) 204.

[12] I. A. Batalin and G. A. Vilkovisky, Phys. Lett. B102 (1981) 27; Phys. Rev. D30 (1983) 2567.

[13] N. Mohammedi, to appear. 